\title{
Mechanical behavior of electrochemically lithiated silicon
}

Lucas A. Berla ${ }^{a,}$, Seok Woo Lee ${ }^{a}$, Yi Cui ${ }^{a, b}$, William D. Nix ${ }^{a}$

${ }^{a}$ Department of Materials Science and Engineering, Stanford University, Stanford, CA 943054034, USA

b Stanford Institute for Materials and Energy Sciences, SLAC National Accelerator Laboratory, 2575 Sand Hill Road, Menlo Park, CA 94025, USA

* Corresponding author. 496 Lomita Mall, Durand Building, Stanford University, Stanford, CA 94305-2205, USA. Tel.: +1 650725 2605; fax; +1 6507254034.

Email: lberla@stanford.edu

\begin{abstract}
The time-independent and time-dependent mechanical behavior of electrochemically lithiated silicon was studied with nanoindentation. As indentation was performed with continuous stiffness measurements during loading and load-hold, new insight into the deformation behavior of lithiated silicon is furnished. Supporting other research, Young's modulus and the hardness of lithiated silicon are found to decline with increasing lithium content. However, the results of this study indicate that Young's modulus of the fully lithiated phase, at $41 \mathrm{GPa}$, is in fact somewhat larger than reported in some other studies. Nanoindentation creep experiments demonstrate that lithiated silicon creeps readily, with the observed viscoplastic flow governed by power law creep with large stress exponents $(>20)$. Flow is thought to occur via local, shear-driven rearrangement at the scale of the $\mathrm{Li}_{15} \mathrm{Si}_{4}$ molecular unit volume. This research emphasizes the importance of incorporating viscoplasticity into lithiation/delithiation models. Additionally, more broadly, the work offers insight into nanoindentation creep methodology.
\end{abstract}

Keywords: lithium ion batteries, lithiation, nanoindentation, silicon 


\section{Introduction}

In light of the growing need to improve energy storage in electronic devices requiring rechargeable battery power, lithium ion battery research has been widely pursued over the past decade [1-3]. Anode design, in particular, has become a focus in the drive for improved battery performance. Silicon, due to its high theoretical specific capacity for electrochemical lithium incorporation, has emerged as one of the most appealing materials to replace conventional graphitic anodes in lithium ion batteries. However, upon lithium insertion silicon undergoes a large volume expansion ( 300\%), which promotes fracture of bulk silicon during lithiation/delithiation cycling and thereby causes capacity fading of silicon anodes. Much progress has recently been made in design of silicon nanostructures that are more resistant to lithiation-induced fracture [4-10]. Although nanostructured silicon shows retarded fracture formation during electrochemical lithium cycling relative to bulk silicon, fracture remains an issue.

The problematic capacity fade exhibited by silicon anodes, brought on by fracture processes during electrochemical cycling, has inspired much research into the details of the lithiation behavior of silicon. Experimental studies have provided insight into the mechanisms by which silicon (both amorphous and crystalline) lithiates [11-14]. Mechanistic knowledge, in turn, has enabled formulation of lithiation models [15-19]. Such models, however, depend strongly upon the inputted mechanical properties of lithiated silicon, and the current understanding of these properties lags that of the lithiation process and its underlying mechanisms.

There have been a few attempts to investigate the mechanical behavior and extract the mechanical properties of lithiated silicon. In three related research projects, an electrochemical 
cell with a silicon anode was constructed, and as the cell was cycled the wafer curvature technique was used to measure the biaxial modulus, yield strength, and fracture toughness of lithiated silicon [20-22]. Pharr et al. also employed a wafer curvature approach to estimate the fracture energy of lithiated silicon [23]. Kushima et al. attempted to determine Young's modulus and the fracture strength of lithiated silicon nanowires by first lithiating crystalline silicon nanowires in a transmission electron microscope (TEM) chamber and then deforming the wires in tension with an in situ TEM piezo device [24]. An x-ray diffraction experiment, wherein a lithiated silicon specimen was subjected to in situ hydrostatic compression, was used by Zeng et al. to establish the bulk modulus of the material [25]. With nanoindentation, Ratchford et al. measured Young's moduli of various equilibrium lithium-silicon alloy phases synthesized at high temperatures $[26,27]$. Hertzberg et al. performed nanoindentation studies, instead on electrochemically lithiated silicon samples, and the authors extracted the modulus and hardness as a function of state-of-charge [28].

Literature on the mechanical properties of lithiated silicon is informative, yet there still exists some uncertainty in the accuracy of the obtained parameters. Specifically, there is substantial variation in the Young's modulus values reported throughout the literature. More important, little is known about the creep behavior of lithiated silicon. Existing models for the process of lithiation of silicon often ignore time-dependent deformation behavior. In view of these remaining issues, we have carried out ex situ nanoindentation studies to characterize the mechanical behavior of lithiated silicon. We have developed a nanoindentation holder that allows nanoindentation to be performed on samples immersed in mineral oil and enables precise lateral positioning of indents via a high-magnification fluid immersion optics system. The utilized nanoindenter possesses continuous stiffness mode (CSM) capabilities, making this work 
the first known lithiated silicon indentation study with CSM-based results. In addition to allowing particularly thorough and reliable hardness and modulus testing, our modified CSM indentation setup gives us the ability to critically assess the nanoindentation creep characteristics of lithiated silicon. We show that Young's modulus of fully lithiated silicon is likely markedly larger than reported in a few previously published studies. Lithiated silicon is found to creep rapidly during constant-load-hold nanoindentation experiments. Using a standard model for thermally activated stress-driven processes, we infer some physical meaning from the observed large power law creep exponents. Our research suggests that models must take into account viscoplastic flow behavior in order to more realistically describe silicon anode lithiation and delithiation.

\section{Experimental}

\subsection{Sample preparation}

Lithiated silicon samples suitable for nanoindentation testing were prepared by depositing silicon films onto molybdenum substrates. The choice to use molybdenum substrates was made in light of molybdenum's lack of reactivity with lithium and its high yield strength. The substrates were cut from a swaged molybdenum rod with wire electrical discharge machining. Residue from the machining process was removed by etching the pieces in a dilute hydrogen peroxide $(5 \%)$ bath, and the resulting scale was removed by etching in concentrated hydrochloric acid. The clean molybdenum discs $(6.3 \mathrm{~mm}$ in diameter and $\sim 480 \mu \mathrm{m}$ thick) were mechanically polished to a mirror finish, and organic mounting debris was removed by immersion into a bath of concentrated sulfuric acid. Finally, the samples were thoroughly cleaned by sonication in solvents (acetone, methanol, and then ethanol), oxide was etched with concentrated sulfuric acid 
and then concentrated hydrochloric acid, and one final ethanol rinse was administered. The above-mentioned, extensive sample preparation measures were followed to reduce surface contamination of the molybdenum, thereby promoting a strong silicon-molybdenum interface and helping to discourage film delamination during lithiation.

Amorphous silicon films were deposited onto the substrates by sputtering with dualtarget AC magnetron sputtering at a constant power of $300 \mathrm{~W}$. Some sputtering batches included an in situ argon ion gun pre-clean to remove native molybdenum oxide prior to deposition; however, pre-etching did not appear to affect the subsequent lithiation behavior of the deposited films. The deposited silicon films were $\sim 1.4 \mu \mathrm{m}$ thick, though there was some variation in the film thickness from sample to sample. An x-ray diffraction scan of a film [Fig. 1(a)]—-showing only diffracted peaks corresponding to the molybdenum substrate - confirmed that the sputtering recipe produced amorphous silicon films.

After film deposition, electrochemical half-cells were assembled. A polyethylene separator was placed between the working electrode (silicon-coated molybdenum samples) and the counter/reference electrode (lithium foil). A solution of $1 \mathrm{M} \mathrm{LiPF}_{6}$ in ethylene carbonate/diethyl carbonate (1:1; Merck) was used as the electrolyte. The pouch cells were hermetically sealed and then removed from the glovebox for electrochemical testing. For samples that were to be fully lithiated (ie. lithiated to $\mathrm{Li}_{15} \mathrm{Si}_{4}$ ), lithiation was performed galvanostatically $\left(7 \mu \mathrm{A}\right.$ current) until the voltage reached $10 \mathrm{mV}$ vs. $\mathrm{Li} / \mathrm{Li}^{+}$, at which the voltage was subsequently held for some time —at least 40 minutes and up to 10 hours—-to further encourage full lithiation. Samples prepared for creep testing ("heavily lithiated" silicon) were perhaps very close to but slightly under the fully lithiated composition. A current of $7 \mu \mathrm{A}$ 
provided lithiation rates for fully lithiated samples near $\mathrm{C} / 50$. Electrochemical data for a fully lithiated sample is shown in Fig. 1(b).

Partial lithiation was also performed galvanostatically at $7 \mu \mathrm{A}$; lithiation was terminated once the total charge transferred reached a specified value corresponding to the desired silicon anode lithium fraction. In order to determine the cutoff charge for partial lithiation, all asdeposited samples were assumed to have the same total mass of amorphous silicon as the first sample subjected to full lithiation. Following lithiation, partially lithiated samples were held under open circuit conditions for at least 10 hours to allow lithium concentration gradients to dissipate by lithium diffusion. Upon completing electrochemical lithiation, the pouch cells were returned to the argon glovebox. The lithiated samples were extracted, rinsed in acetonitrile, and then mounted onto aluminum pucks using Crystalbond 509 adhesive. Inside the glovebox, the mounted samples were placed into vials filled with paraffin oil prior to being transferred to the nanoindenter system. Samples were kept immersed in paraffin oil during nanoindentation, so the air-sensitive lithium-containing samples were never exposed to ambient air.

Although sample preparation procedures were designed to limit film delamination during lithiation, delamination was not entirely avoided. During lithiation, film blistering developed in both fully lithiated and partially lithiated films. Such blistering is depicted in Fig. 2, which is a scanning electron microscope image ( $52^{\circ}$ stage tilt) of an amorphous silicon film on molybdenum that was fully lithiated potentiostatically. As performing nanoindentation on blistered film regions would provide uninterpretable data, it was necessary to selectively indent the film between blisters, where the film remained adhered to the substrate.

\subsection{Nanoindentation}


Because lithium rapidly oxidizes in humid air, nanoindentation was performed on samples immersed in a protective paraffin oil bath. All indentation experiments were carried out with an Agilent Technologies Nanoindenter XP using a diamond Berkovich tip. In addition to its continuous stiffness measurement (CSM) capabilities, this instrument has a high load limit ( 500 $\mathrm{mN}$ ) useful for making deep indentations not greatly influenced by sample roughness or surface oxide. However, as the indentation axis travel range of the indenter head is only $1500 \mu \mathrm{m}$, it can be challenging to perform nanoindentation on samples immersed deep (several millimeters) in fluids. One approach is to mount a small fluid cell, at the bottom of which is the sample, onto a stage that allows tunable movement in the vertical direction (along the indentation axis). With this feature, the sample, beneath the indenter tip, could be raised until it is only a small distance (less than the indenter head travel range) from the tip. A consequence of the need to adjust the sample's vertical position — when the indenter tip is directly above the specified indent location but not yet touching the sample surface- - is that lateral precision in indent location designation can be lost. As a result, this method might not allow us to dependably place indents on the film area between blisters. We thus constructed a new sample holder, with a fluid reservoir large enough to enclose both the indenter tip and the optics ( $\times 100$ oil-immersion objective). Proper initial mounting of the indentation specimen eliminated the need to make subsequent changes to the vertical position of the sample, and thus the resolution in indent lateral position was determined by the resolution of the instrument's precision (x,y)-stage motors.

All hardness- and modulus-extraction nanoindentation experiments were performed by continuously monitoring the sample stiffness with the CSM technique ( $2 \mathrm{~nm}$ oscillation) while loading at a constant nominal indentation strain rate of $0.05 \mathrm{~s}^{-1}$. Thermal drift was recorded following each indentation test and then subtracted from the raw data. For nanoindentation creep 
tests, both quasi-static (no dynamic oscillation) and continuous stiffness measurement (4 nm oscillation) creep tests were implemented. Creep studies on lithiated silicon were performed using constant-load-hold tests. The indenter was ramped up to the peak load in $10 \mathrm{~s}$, and the peak load was held at $15 \mathrm{mN}$ for $300 \mathrm{~s}$ (quasi-static) or $\sim 16.5 \mathrm{mN}$ for $600 \mathrm{~s}$ (CSM). Unlithiated, amorphous silicon was also probed via nanoindentation creep testing. In making comparisons between the creep rates of amorphous silicon and those of lithiated silicon, it was most informative to execute the different creep tests at a similar effective indentation pressure. To achieve this, because amorphous silicon has a much greater hardness than lithiated silicon, nanoindentation creep tests on amorphous silicon were performed after partially unloading the indenter from the peak indentation load. The indenter was loaded to a peak load of $\sim 15.5 \mathrm{mN}$ in $10 \mathrm{~s}$ after which another $10 \mathrm{~s}$ were taken to reduce the load until the calculated peak oscillatory pressure under the indenter was $\sim 1.7 \mathrm{GPa}$. The load at this reduced pressure was held for $600 \mathrm{~s}$ as CSM creep testing was performed. Some partially unloaded CSM creep tests were also made on lithiated silicon, though the peak load $(\sim 16 \mathrm{mN})$ was held for $20 \mathrm{~s}$, and the final reduced contact pressure at which creep testing was performed was $\sim 520 \mathrm{MPa}(5.8 \mathrm{mN})$. Finally, some quasi-static indentation recovery experiments were made; lithiated silicon was subjected to creep testing for $600 \mathrm{~s}(15 \mathrm{mN})$ after which the indenter was unloaded to $0.68 \mathrm{mN}$ for recovery monitoring (600 s). In all analyses, to convert the empirically measured reduced moduli to Young's moduli, it was assumed that Poisson's ratio for lithiated silicon follows a linear rule of mixtures between that of amorphous silicon $(v=0.22)$ and that of pure lithium $(v=0.36)$.

\section{Results and Discussion}

\subsection{Modulus and hardness evaluation}


Various reference materials were indented first in air and then while immersed in paraffin oil, and the findings confirmed that immersion in the oil does not notably affect continuous stiffness measurement (CSM) indentation results. Following these preliminary tests, six samples were studied with basic CSM nanoindentation for modulus and hardness measurement. Specifically, we probed one as-deposited amorphous silicon film $\left(x_{L i}=0\right)$, three partially lithiated silicon films $\left(x_{L i}=0.52, x_{L i}=0.64\right.$, and $\left.x_{L i}=0.71\right)$, one fully lithiated silicon film $\left(x_{L i}=0.79\right)$, and one square of bulk lithium foil $\left(x_{L i}=1\right)$. Each of the silicon-containing specimens was indented in twenty five separate locations, although only fourteen indents were made into the bulk lithium sample. For each sample dataset, obvious outlying data were disregarded, and the resulting refined datasets were averaged.

The data-averaging process is represented in Fig. 3(a), which gives modulus data from twenty four indents made into fully lithiated silicon along with the averaged result. Fig. 3(b) shows, for each sample, the averaged Young's modulus plotted versus contact depth $\left(h_{c}\right)$. Since the indented material is in the form of relatively thin, compliant films on stiff molybdenum substrates, the measured moduli are rising composite moduli that reflect both the film and the substrate properties. Film moduli, deconvoluted from the elastic properties of the substrate, are extracted from the curves presented in Fig. 3(b) by making a linear fit to the data in the region where the contact depth is between $5 \%$ and $15 \%$ of the film thickness; the film modulus is taken to be the extrapolated vertical-axis intercept of the fit line. The film thickness increase that accompanies lithiation is considered in determining the appropriate fitting region. Fig. 3(c) illustrates application of this extrapolation procedure to fully lithiated silicon data. Of course, roughness influences the measured modulus-versus-depth curves at very small contact depths. 
However, the minimum contact depth for our fitting range is sufficiently large that the employed modulus extraction procedure is not affected by surface roughness.

In Fig. 3(d), the composite indentation hardness, after data averaging, is plotted as a function of contact depth. At small depths, the data are greatly influenced by roughness and, potentially, the presence of surface oxide. At large depths of indentation, the measured hardness either increases or decreases, with the behavior depending on the hardness of the indented film relative to the hardness of the molybdenum substrate $(\sim 3.5 \mathrm{GPa})$. The hard, low-lithium-content films $\left(x_{L i}=0, x_{L i}=0.52\right)$ show a decreasing composite hardness following substrate yielding. On the other hand, the composite hardness of the softer, more heavily lithiated films $\left(x_{L i}=0.64\right.$, $\left.x_{L i}=0.71, x_{L i}=0.79\right)$ rises at large depths. For all samples, however, there is a relatively flat region at intermediate depths - a valley, plateau, or shelf-that corresponds to local deformation of the film with little substrate influence on plasticity. The width of this region increases with degree of lithiation as the film thickness grows. The hardness of the film material, independent of the substrate, is taken to be the average value of the composite film/substrate hardness in this intermediate flat zone.

From Figs. 3(b) and 3(d), it appears that increasing the lithium content in the films decreases both the hardness and the modulus of the film. This effect is shown more clearly in Fig. 4, which plots the extracted film modulus [4(a)] and the extracted film hardness [4(b)] as a function of lithium fraction. The data stemming from our herein-described experiments ("Berla et al.") are designated with filled triangular symbols. Included in Fig. 3 are all available data points from existing nanoindentation-based research on the mechanical properties of lithiated silicon. The modulus values we acquire, with CSM-based nanoindentation, match expectations in the limits of amorphous silicon (104.6 GPa) and pure, metallic lithium (8.0 GPa). At 
intermediate compositions, Fig. 4(a) reveals the modulus of lithiated silicon as being characterized by two seemingly distinct regions. At low lithium concentrations, increasing the lithium content appears to only gradually reduce Young's modulus. Adding 52\% (atomic percent) lithium to amorphous silicon only produces a 13\% reduction in Young's modulus. In the high-lithium-content $\left(x_{L i}>0.52\right)$ region, however, the modulus, exhibiting linear-rule-of-mixtures behavior, falls much more steeply as lithium content is increased.

It is evident from Fig. 4(a) that both our work and that of Hertzberg et al. suggest that lithium incorporation into silicon has an elastic softening effect [28]. Despite these similarities in overall trend, there is a significant discrepancy between the Young's modulus of fully lithiated silicon $\left(x_{L i}=0.79\right)$ measured in our work and that reported by Hertzberg et al. The latter found the fully lithiated phase to have a Young's modulus of $12 \mathrm{GPa}$, which is more than three times smaller than the Young's modulus we infer (41 GPa). Kushima et al., with lithiated silicon nanowire tension testing, also measured a low Young's modulus of 7.9 GPa for the fully lithiated phase [24]. For comparison, the Young's modulus of polycrystalline lithium found in the literature is $\sim 8 \mathrm{GPa}[29,30]$. It is surprising that the moduli of fully lithiated silicon measured by Kushima et al. and Hertzberg et al. are equal to or not much larger than the modulus of pure lithium. As lithium-silicon intermetallic phases melt at temperatures much higher than the melting point of pure lithium, one might expect the modulus of fully lithiated silicon to be appreciably higher than that of pure lithium. The Young's modulus of fully lithiated silicon (41 GPa) that we extract in our indentation study might thus be more reasonable.

There exists a noteworthy body of literature on simulations of mechanical properties of lithiated silicon [31-37]. Unfortunately, though, the range in the fully lithiated silicon moduli predicted by the various simulations is rather large. Kushima et al. predicted with quantum 
mechanical ab initio simulations a fully lithiated silicon Young's modulus of $33.5 \mathrm{GPa}$ [24]. Using DFT simulations, Shenoy et al. calculated Young's modulus of a fully lithiated silicon polycrystal to be about $50 \mathrm{GPa}$ [31]. Fan et al., on the other hand, employed molecular dynamics simulations with a reactive force field to find a fully lithiated silicon (amorphous) Young's modulus of only $12 \mathrm{GPa}$ [32]. In light of this variance in moduli extracted from simulations, the present simulation literature does not provide great clarity in understanding the realistic magnitude of the Young's modulus of fully lithiated silicon. Some experimental work, on the other hand, does lend support to the high modulus that we find. A very recent wafer curvature study implies that Young's modulus of lithiated silicon is $50 \pm 15 \mathrm{GPa}$ [38]. It is interesting to note that the Young's modulus of fully lithiated silicon resulting from our indentation experiments nearly matches the modulus of the thermodynamically stable lithiated $\mathrm{Li}_{22} \mathrm{Si}_{5}$ phase — plus symbol at $x_{L i}=0.81$ in Fig. 3(a)—determined by Ratchford et al. [26,27]

Fig. 4(b) compares the hardness-versus composition results from our research with those of Hertzberg et al. [28] Both our data and the data of the other authors illustrate that silicon is progressively softened by adding lithium. However, for unlithiated silicon, there is a prominent disparity in the hardness measured in our work compared to that reported by Hertzberg et al. Our research implies a pure, amorphous silicon hardness of $10 \mathrm{GPa}$, which falls within the range (8.8$11.3 \mathrm{GPa}$ ) reported in literature [39,40]. Hertzberg et al. reported that the hardness of unlithiated silicon is only $5 \mathrm{GPa}$, which is a surprisingly low number. The most likely explanation for the low hardness measured by Hertzberg et al. is the existence of a rather open structure in the asdeposited silicon films and, perhaps, porosity at the nanoscale. It follows that, since their silicon films prior to lithiation are unusually soft, the phases with low lithium content are also especially soft. Interestingly, for more heavily lithiated silicon films, the hardnesses extracted by Hertzberg 
et al. match up quite closely with those found in our studies. We find the fully lithiated phase to have a hardness of $1.3 \mathrm{GPa}$, and Hertzberg et al. claimed a hardness for this phase of $1.5 \mathrm{GPa}$. This outcome-insensitivity of the plastic response of fully lithiated silicon to nanoporosity in the initial silicon film - is hypothesized to occur by way of lithiation-induced volume dilation producing densification of material and porosity elimination. Finally, we note that, according to the well-known Tabor relationship, the concluded hardness (1.3 GPa) signals a yield strength of fully lithiated silicon around $\sim 430 \mathrm{MPa}$.

\subsection{Viscoplastic creep}

Upon initiation of our research, little was known about the time-dependent deformation response of lithiated silicon. Our initial CSM nanoindentation creep studies, motivated by an absence of research on creep of lithiated silicon, are shown in Fig. 5. Fig. 5 depicts, for both amorphous silicon and heavily lithiated silicon, the normalized stiffness change plotted versus time for constant-load-hold creep tests. Though the horizontal axis is labeled "Time", it in fact represents the time elapsed $(\Delta t)$ since thirty seconds after the commencement of the creep load hold. The first thirty seconds of the load-hold segment are disregarded in our creep analyses because the CSM system is somewhat unstable during this initial period. This convention is utilized throughout the remainder of the manuscript, although for quasi-static creep tests, wherein instrument stabilization issues are not as significant, the reference time $(\Delta t=0)$ corresponds to only two seconds after the beginning of the load hold. The portrayed data are acquired by averaging the stiffness-versus-time data from distinct creep tests (twenty tests for amorphous silicon and nine tests for lithiated silicon). The normalized stiffness change, which is given as a percentage in Fig. 5, is defined as $\Delta S / S_{o}=\left(S-S_{o}\right) / S_{o}$, where $S$ is the current contact 
stiffness, and $S_{o}$ is the stiffness measured at the start of the creep analysis. This parameter provides a measure of the creep strain as the indenter sinks into the sample.

Particularly during the early stages of the load hold, the curve slope in Fig. 5-a reflection of the creep strain rate-is much larger for lithiated silicon than it is for amorphous silicon. Moreover, the terminal normalized stiffness change for lithiated silicon is nearly a factor of seven larger than for amorphous silicon. Fig. 5 very clearly illustrates that, at the same indentation pressure, lithiated silicon creeps much more readily than does unlithiated amorphous silicon. Lithium, of course, is a small, mobile atomic species, and metallic lithium is known to behave viscoplastically [30]. Hence, it seems reasonable that lithiated silicon, with its high lithium content and its weakened $\mathrm{Li}-\mathrm{Si}$ bonding relative to covalent silicon bonding, would display such creep behavior. Our unambiguous observation of room-temperature creep in lithiated silicon underscores the inadequacy in current lithiation models that do not account for viscoplastic flow and consequent stress relaxation. More generally, though, this finding provides a stimulus for more deeply understanding viscoplastic flow of lithiated silicon.

In our quasi-static nanoindentation creep analysis, we calculate the effective indentation strain rate $\dot{\varepsilon}$ as $\dot{\varepsilon}=\dot{h} / h$,

where $h$ is the indenter displacement, and $\vec{h}$ represents the instantaneous indenter displacement rate. The displacement rate is calculated by fitting the creep $h$-versus- $\Delta t$ curve with $h(\Delta t)=C_{1}\left(\Delta t-C_{2}\right)^{c_{\mathrm{I}}}$

and then analytically differentiating the fitted function with respect to $\Delta t$. In Eq. (2), $C_{1}, C_{2}$, and $C_{3}$ are all fitting constants. As will be justified in a subsequent section, this particular function is 
used due to its underlying consistency with indentation of a power law creeping material. The stress $(p)$ —or effective pressure beneath the indenter-is defined as $p=F / A_{c}$,

where $F$ is the applied indentation load, and $A_{c}$ is the indentation contact area. To calculate the contact areas during creep, the quasi-static indenter displacements are converted to contact depths by separately determining the $h_{c}(h)$ relationship for lithiated silicon with CSM indentation. The contact areas are then calculated with the calibrated tip area function $A_{c}\left(h_{c}\right)$. Finally, as we expect the observed lithiated silicon creep to be viscoplastic in nature, we characterize the flow with a standard power law creep constitutive law $\dot{\varepsilon}=B p^{n}$,

where $B$ is a constant, and $n$ is the creep stress exponent. On a plot of $\log (\dot{\varepsilon})$ versus $\log (p)$ the stress exponent is simply extracted as the slope of the curve.

Fig. 6(a) depicts the creep analysis employed on one representative creep test. In the quasi-static indentation creep analyses, data obtained more than $22 \mathrm{~s}$ after load hold initiation are not considered. At long times, the creep displacement rate, previously much greater in magnitude than the thermal drift rate of the sample, becomes small enough that thermal drift can no longer be disregarded. The difficulty in properly and precisely removing thermal drift from indentation data renders the long-time quasi-static creep data uninterpretable with the employed analysis. Fig. 6(a) confirms that Eq. (2) provides close fits to the creep data. Included in Fig. 6(b) are analyzed $\dot{\varepsilon}$-versus- $p$ data $\left(\log _{10}-\log _{10}\right.$ scale) for all nine (distinct sample locations) of the performed quasi-static creep tests. Each test displays power-law behavior with a constant stress exponent throughout the analyzed time range. The individual curves do not fall perfectly on top one another likely due to the difficulty in defining, and assessing, the pressure beneath the 
indenter. However, the mismatch is small, and, more importantly, the evolution of the creep behavior appears very consistent. The average stress exponent is determined to be $n=24.8 \pm 1.7$, with the small standard deviation conveying the stated behavioral consistency.

The documented quasi-static analysis, which is necessarily confined to short times due to challenges associated with thermal drift, of course bears the possibility of providing only a limited understanding of the creep behavior of lithiated silicon. Creep studies limited to such short times, for instance, may describe primary creep instead of secondary creep. In response to such concerns, we have supplemented quasi-static nanoindentation creep testing with CSMbased nanoindentation creep experiments that permit creep analysis to extend to much longer times. In the method of analysis applied for CSM-based indentation creep experiments, the indentation depths were calculated directly from the measured harmonic contact stiffnesses following Maier et al. [41] In the approach proposed by Maier et al., stiffnesses are converted into contact areas using the well-known Sneddon equation. Contact areas are next transformed into contact depths by numerical inversion of the known calibrated indenter tip shape function. Finally, from the contact depths, indenter displacements are computed using the well-known Oliver and Pharr relationship. The determined displacement values are unaffected by thermal drift, and thus this analysis can be used to assess indentation creep at long times when the creep displacement rate is comparable to or even smaller than the thermal drift rate. Upon obtaining the drift-free indenter displacements, the subsequent steps in the analysis are analogous to those outlined previously for quasi-static indentation creep analysis.

After averaging the raw stiffness-versus-time data for all of the analyzed creep tests (see Appendix A), the data are transformed into a displacement-versus-time curve using the method of Maier et al. In executing the Maier analysis, the sample reduced modulus is assumed to be 
$76.5 \mathrm{GPa}$, which is measured during the beginning of the load-hold segment. Note that this modulus is larger than that provided for fully lithiated silicon in Fig. 4(a), as the modulus used in this analysis is a composite modulus that reflects both the elastic properties of the film and those of the underlying substrate. The resulting displacement-versus-time data, included in Fig. 7(a), is fit well with Eq. (2). Similarly, the pressure-versus-time data is fit with a power-law function, with form analogous to Eq. (2), for the sake of data smoothing. Completion of the analysis provides $\dot{\varepsilon}$-versus- $p$ data, which is presented in a $\log _{10}-\log _{10}$-scale plot in Fig. $7(\mathrm{~b})$. The power law creep stress exponent that results from the analysis is reasonably large at $n=20.8$, which compares to value inferred from the quasi-static creep analysis $(n=24.8 \pm 1.7)$. Evident from Fig 7(b) is the advantage of CSM-based indentation creep studies; the ability to exploit creep data at long times allows us to probe strain rates that are smaller- even much smaller- than those examined in the quasi-static creep tests [Fig. 6(b)]. On the other hand, since the CSM system is not fully stable during the first thirty seconds of the load hold, the high-strain-rate region can only be captured and assessed with quasi-static creep testing. In this way, the quasistatic creep testing and the CSM creep testing complement each other. Together they offer a view into the creep behavior of lithiated silicon over a rather wide range of strain rates.

An additional set of CSM-based lithiated silicon creep tests were carried out by imposing the load hold after partially unloading the indenter from the peak load. This partial unloading made it possible to probe the creep behavior at low indentation pressures ( $<520 \mathrm{MPa}$ ). The creep analysis, which assumes a reduced sample modulus of $56.6 \mathrm{GPa}$, is represented in Fig. 7(c). The analysis outcome $(\dot{\varepsilon}$-versus- $p)$ is plotted in $\log _{10}-\log _{10}$ scale in Fig. $7(\mathrm{~d})$. Once again, the stress exponent measured is large $(n=29.5)$. This stress exponent is even larger than those obtained through our standard quasi-static $(n=24.8 \pm 1.7)$ and CSM indentation creep experiments 
$(n=20.8)$. It is now apparent that the presented indentation creep research, regardless of approach, consistently indicates that the creep behavior of lithiated silicon is characterized by very large power law creep stress exponents-always greater than 20 in magnitude.

In reviewing the collection of lithiated silicon creep results communicated in this manuscript, it is worth giving further attention to several points. We have studied creep behavior across a range in strain rates by utilizing different experimental techniques (QSM, fully loaded CSM, partially unloaded CSM), but the various $\dot{\varepsilon}$-versus- $p$ curves found do not fall on the same line when shown together in one $\log _{10}-\log _{10}$-scale plot. As mentioned previously, such mismatch might possibly develop due to the inherent difficulty in defining the indentation pressure. The pressure calculation may be affected by growth of the surface oxide film. In fact, progressive growth of the surface oxide film seems to decrease the measured film hardness and thus the measured indentation pressure. Additionally, differences may arise due to differing atomic structures of the various lithiated films. The sample that is tested with standard QSM- and CSMbased creep testing [Figs. 6 and $7(a, b)]$ is anticipated to be amorphous $\mathrm{Li}_{15} \mathrm{Si}_{4}$. The initial thickness of the as-deposited amorphous silicon film $(\sim 1.4 \mu \mathrm{m})$ is smaller than the critical initial film thickness (Hatchard and Dahn [42]) above which silicon films typically crystallize, upon electrochemical lithiation, into metastable polycrystalline $\mathrm{Li}_{15} \mathrm{Si}_{4}$. It is possible that the film studied with partially unloaded creep testing [Fig. 7(c,d)] possesses some degree of crystallinity; this film, prior to lithiation, was slightly thicker than all others described in this manuscript. Although the data produced with different techniques fails to map onto a single line, the viscoplastic flow evolves in a consistent manner regardless of the testing method used. That is, we always find power law creep behavior with large stress exponents. Consistency in flow 
evolution is a consequential concept, as it implies a single mechanism—or single set of mechanisms - is responsible for all of the observed viscoplasticity.

\subsection{Local rearrangement during creep}

The stress exponent, when small, can give insight into the mechanisms that control creep. For example, power law creep with an exponent of 1,2, and 3 can be justified as describing creep by atomic diffusion, grain boundary sliding, and dislocation climb, respectively. However, it is not immediately clear what such large exponents (>20) reveal to us about the physical processes responsible for creep of lithiated silicon. To probe this issue, it is illuminative to make use of a simple model. For a creep process occurring by a thermally activated, shear stress-driven flow mechanism, such that

$\dot{\varepsilon}=\dot{\varepsilon}_{0} \exp \left(-\frac{\Delta G(\tau)}{k_{B} T}\right)$,

the activation volume $\Delta V^{*}$ can be shown to be

$\Delta V^{*}=n \cdot k_{B} T / \tau$,

where $n, k_{B}, T, \tau$ are the power law creep exponent, Boltzmann constant, temperature, and shear stress, respectively. The model, commonly used to describe dislocation-driven processes, may also be applicable to processes transpiring in amorphous solids [43], such as our lithiated silicon sample with anticipated amorphous structure [Figs. 6 and 7(a,b)]. Considering the Mises yield criteria $(\tau=\sigma / \sqrt{3})$ and the Tabor relationship ( $\sigma=p / 3)$, Eq. (6) may be converted to a form practical for nanoindentation:

$\Delta V^{*}=n \cdot 3 \sqrt{3} k_{B} T / p$

With Eq. (7), the activation volume can be calculated with knowledge of the stress exponent and the applied indentation pressure. 
Inspecting the room-temperature quasi-static creep data [Fig. 6(b)], the calculated activation volume varies between $\Delta V^{*}=361 \pm 20.8 \AA^{3}(t=0 \mathrm{~s})$ and $\Delta V^{*}=395 \pm 21.5 \AA^{3}$ $(t=20 \mathrm{~s})$. By comparison, assuming that the density of unlithiated, amorphous silicon is $\rho=2.3 \mathrm{~g} / \mathrm{cm}^{3}$ and the volume expansion upon full lithiation is given by $V_{f} / V_{i}=3.7$, the volume of a molecular unit of $\mathrm{Li}_{15} \mathrm{Si}_{4}$ is determined to be $V\left(\mathrm{Li}_{15} \mathrm{Si}_{4}\right)=300 \AA^{3}$. Thus, the power law behavior captured by our creep experiments, with its characteristic large (>20) stress exponent, is consistent with local rearrangement on the scale of a molecular unit of $\mathrm{Li}_{15} \mathrm{Si}_{4}$. The calculation draws a clear distinction between the creep behavior observed in this research and that which would be expected from a sample with creep controlled by diffusion of atomic species, wherein the magnitude of the activation volume should approach an atomic volume.

\subsection{Recent research on creep of lithiated silicon}

Quite recently, since commencement of our research, another research group published a study on the creep properties of lithiated silicon. Boles et al. conducted very difficult nanowire tension testing experiments, wherein nanowires were lithiated in situ prior to mechanical testing in an electron microscope chamber [44]. The authors' creep investigation consisted of testing three individual lithiated silicon nanowires, which prior to lithiation had diameters between 80 $\mathrm{nm}$ and $150 \mathrm{~nm}$. The authors were not able to determine if the as-lithiated nanowire structure was amorphous or crystalline. For each nanowire, the steady state strain rate was measured at various (five to eight) tensile stress levels. Plots of the strain rate versus the applied wire stress reveal linear viscous creep behavior (ie. stress exponent $n=1$ ) at low stresses and, purportedly, occasional power-law breakdown at large stresses. 
The linear viscous flow reported by Boles et al. stands in stark contrast with the largeexponent power law behavior indicated by our indentation creep experiments. It is difficult to pinpoint a basis for the discrepancy. As with nearly any nanoindentation result that is subject to scrutiny, we are compelled to question whether pileup around the indenter may have skewed our observed results. Material pileup, progressing during creep testing, when unaccounted for would lead to underestimation of the true indentation pressure relaxation. Since the stress exponent is measured as the change in strain rate divided by the pressure reduction, failure to account for pileup could thus give unrealistically large stress exponents. However, pileup does not seem to be an issue in our research. The CSM-based indentation creep data, which is produced with the Maier et al. method of analysis [41], is not influenced by potential pileup since all relevant data are computed directly from the recorded contact stiffnesses. Because the quasi-static creep results, which do not effectively account for potential pileup, make similar predictions as the CSM-based results, it appears that pileup is not likely to be a factor in any of our creep experiments. It is possible that the difference in creep behavior between our work and that of Boles et al. could arise from differences in sample lithiation methods. Samples in our work were lithiated by controlled galvanostatic lithiation with a potential limit $\left(10 \mathrm{mV}\right.$ vs. $\left.\mathrm{Li} / \mathrm{Li}^{+}\right)$, whereas Boles et al. lithiated the silicon nanowires by simply contacting the nanowires with metallic lithium. In any case, it is important that an effort be made in the future to identify the origin of the inconsistency between these two creep reports. Another manuscript just published this year offers some confirmation for large stress exponents [38]. Through some wafer curvature experiments, the authors discover lithiated silicon to be somewhat strain-rate insensitive with large stress exponents around $n=50$. These findings, consistent with ours, do not agree with the linear viscous, strain rate sensitive flow behavior reported by Boles et al. 
One other lithiated silicon creep mechanism worth addressing is the seemingly appealing conception of time-dependent flow by lithium atom diffusion through an elastic silicon network. In explaining indentation creep with this model, the diffusional flow of mobile lithium atoms, away from the indenter, is driven by chemical potential gradients, which are in turn produced by the pressure gradients associated with the indentation stress field. Such a process would exhibit extensive recovery upon load reduction, as lithium would diffuse back into the lithium-depleted regions directly beneath the indenter. However, indentation recovery tests (Fig. 8) show little displacement recovery [Fig. 8(b)] following creep, so it seems that this diffusional mechanism is not predominantly responsible for the observed time-dependent deformation behavior. Creep of lithiated silicon looks to be primarily governed by irreversible viscoplasticity. Nonetheless, keeping in mind that slight recovery is seen in Fig. 8(b), it is certainly possible that reversible diffusional flow still makes minor contributions to the overall indentation creep behavior.

\subsection{A note on indentation creep analysis}

A crucial step in nanoindentation creep analyses is determination of the strain rates. This calculation, described previously, requires knowledge of the displacement rates, which are computed by fitting to creep $h(t)$ curves and differentiating the fit functions. Selection of the functional form used to fit the data is an important topic. A quick survey of indentation creep literature shows widespread use of empirical fitting functions. Li and Ngan proposed an empirical function given by

$h(t)=h_{\circ}+a\left(t-t_{0}\right)^{b}+k t$,

where $h_{o}, a, t_{o}, b$, and $k$ are all fitting constants [45]. Many others follow Li and Ngan and adopt Eq. (8) in indentation creep analyses [46-52]. Since Eq. (8) is empirical in nature, use of 
the equation in indentation creep analyses must be done with caution. The function, with its numerous (five) fitting parameters, may still fit creep $h(t)$ curves well. However, Eq. (8), fit to creep $h(t)$ data, may also embody unphysical behavior that can ultimately engender extraction of unrealistic creep exponents.

In our analyses, we seek to use a fitting function that is consistent with the physics of indentation of a power law creeping solid. Taking similar initial steps as Su et al. [53], the appropriate function can be ascertained by substituting the displacement-dependencies of $\dot{\varepsilon}$ [Eq. (1)] and $p\left[F /\left(C h^{2}\right)\right]$ into the governing power law [Eq. (4)]. Integration of the result, while making sure to implement the proper integration limits, yields the following:

$h(t)=\left(\frac{2 n B F^{n}}{c^{n}}\left(t-t_{o}\right)+h_{o}^{2 n}\right)^{\frac{1}{2 n}}$

The parameters $t_{o}$ and $h_{o}$ are the time and indenter displacement, respectively, at the beginning of the analyzed portion of the constant-load hold. Upon rearranging and renaming constants, Eq. (9) reduces to

$h(t)=C_{1}\left(t-t_{0}-C_{2}\right)^{C_{s}}=C_{1}\left(\Delta t-C_{2}\right)^{C_{s}}$,

which is the same as Eq. (2). It should now be clear that we select Eq. (2), with its particular form, for our creep analyses due to its physical relevance. Often it is difficult to judge the quality of different functional fits to data. This is particularly true when evaluating CSM stiffness-based creep data, which is typically somewhat noisy. Use of Eq. (2) promises us, for a given viscoplastic sample, the stress exponent that best characterizes the power law creep behavior of the sample. Due to its lack of a physical basis, Eq. (8), when used to extract stress exponents, cannot offer such assurance that the predicted stress exponents will describe the actual power law creep behavior of the sample. 
The above-derived form of Eq. (10)—or, equivalently, Eq. (2)—is suitable for performing fits to ideal creep data that is unaffected by thermal drift. None of the data we have analyzed to this point are significantly influenced by thermal drift, as data were either obtained directly from contact stiffnesses $(\mathrm{CSM})$ or from small-time $(t<20 \mathrm{~s})$ quasi-static creep data, wherein creep-driven displacement rates are much greater than thermal drift rates. Analysis of data affected by drift is discussed in Appendix B.

\section{Summary and Conclusions}

A nanoindentation holder, permitting fluid-immersion indentation with high lateral precision has been developed. The equipment has permitted continuous stiffness measurement (CSM) nanoindentation to be conducted on electrochemically lithiated samples immersed in a protective paraffin oil bath. This research, the first study of lithiated silicon with CSM-based nanoindentation, gives new insight into the time-independent and time-dependent deformation behavior of lithiated silicon.

The modulus and hardness of lithium/silicon alloy films of various compositions have been studied by nanoindentation. The results clearly demonstrate that the addition of lithium to silicon has an effect of elastic and plastic softening, as both the modulus and hardness drop with increasing lithium concentration. Young's modulus of fully lithiated silicon is found to be 41 $\mathrm{GPa}$, which is more than a factor of three larger than that reported by Hertzberg et al. [28] Our research reveals the hardness of fully lithiated silicon to be $1.3 \mathrm{GPa}$, a number that is consistent with the research of Hertzberg et al. The hardness, assuming the Tabor relationship $H=3 \sigma$, indicates that the yield strength of fully lithiated silicon, at the strain rate studied, may be around $\sim 430 \mathrm{MPa}$. 
Nanoindentation creep experiments have also been made on unlithiated, amorphous silicon as well as on heavily lithiated silicon samples. In an effort to explore the creep response of lithiated silicon across several orders of magnitude in strain rate, both quasi-static and CSMbased constant-load-hold tests have been performed. Creep is shown to be very active in lithiated silicon relative to unlithiated silicon. The findings from all of our creep studies are described by a power law creep constitutive law with large stress exponents $\left(n_{\text {quasi }}=24.8\right.$ and $n_{C S M}=20.8$ ). Taking creep to act through stress-driven, thermally activated processes, the calculated stress exponents predict an activation volume of $361-395 \AA^{3}$, which is comparable to the volume of a molecular unit of $\mathrm{Li}_{15} \mathrm{Si}_{4}\left(300 \AA^{3}\right)$. Thus, the measured viscoplastic flow is believed to occur by a mechanism of local, concerted rearrangement of small atomic clusters rather than individual atoms. More research on creep of lithiated silicon is needed to identify the cause of the discrepancy between our power law observations and those of Boles et al. [44] illustrating linear viscous behavior in tension.

The results communicated herein provide more clarity to and further our understanding of the mechanical properties and deformation behavior of lithiated silicon. Most significantly, the importance of viscoplastic creep in lithiated silicon is demonstrated. The work reveals that creep and stress relaxation must be accounted for in lithiation models, and methods for representing this behavior are described in this manuscript. New lithiation/delithiation cycling models accounting for these observations should provide more realistic insight into the cycling behavior of silicon anodes. Finally, some general notes on nanoindentation creep analyses are made.

\section{Acknowledgements}


L.A.B. and W.D.N. are grateful for support from the Office of Science, Office of Basic Energy Sciences, of the U.S. Department of Energy under Contract No. DE-FG02-04ER46163. S.W.L. and Y.C. acknowledge support from the U.S. Department of Energy, Office of Basic Energy Sciences, Division of Materials Sciences and Engineering under Contract No. DE-AC0276SF00515 through the SLAC National Accelerator Laboratory LDRD project and the Assistant Secretary for Energy Efficiency and Renewable Energy, Office of Vehicle Technologies of the U.S. Department of Energy under Contract No. DE-AC02-05CH11231, Subcontract No. 6951379 under the Batteries for Advanced Transportation Technologies Program.

\section{Appendix A. Data averaging for CSM-based creep testing}

If the method of Maier et al. [41] is used to analyze CSM indentation creep data from a single test, the notable noise in the harmonic contact stiffness data is inevitably echoed in the stiffness-derived creep displacements, making creep analysis potentially difficult. Noise in the final displacement-versus-time data can be reduced by averaging the raw stiffness-versus-time creep data, from multiple tests, prior to embarking upon the Maier analysis. However, close inspection of stiffness-versus-time datasets from separate creep tests on lithiated silicon reveals that they tend to drift directly upward toward higher stiffness values with each subsequent test. This effect is thought to originate from slow growth of the surface oxide layer over time. It does not make sense to average stiffness-versus-time datasets that are offset noticeably, such as that of the first creep test and that of the final creep test. The first handful of creep tests that were performed, however, provide stiffness-versus-time datasets with no obvious separation. Accordingly, consideration was only given to tests made prior to the point at which dataset shift becomes visually noticeable; these tests were averaged. Of the seventeen CSM-based indentation 
creep tests made at peak load on the heavily lithiated silicon sample [Figs. 7(a,b)], only the first nine were used for stiffness-versus-time data averaging. For partially unloaded creep testing [Figs. 7(c,d)], the first four tests were used for stiffness-versus-time data averaging.

\section{Appendix B. Thermal drift during quasi-static creep testing}

In evaluating long-time quasi-static indentation creep data, wherein the thermal drift rate is comparable to the creep displacement rate, it is important that the creep analysis accounts for thermal drift. Fig. B.1(a) depicts a plot of the raw displacement versus time from a quasi-static load-hold. The data are shown for not only the first twenty seconds but for the entire duration (300 s) of the load hold. The plotted raw displacements reflect actual materials creep response combined with some unknown amount of drift, which may be linear with time so long as the analyzed time range is not too large. Such drift must be accounted for when analyzing long-time quasi-static creep data, and — considering the findings of Peykov et al. [52] — we do so by incorporating an additional linear fitting term into Eq. (2):

$h(\Delta t)=C_{1}\left(\Delta t-C_{2}\right)^{C_{\mathrm{g}}}+C_{4} \Delta t$

Eq. (B.1) provides a very close fit to the raw data [Fig. B.1(b)] over the entire span in time (300 s). Fits made over this same time range using Eq. (2), without inclusion of the linear term, are noticeably worse.

Fitting the raw $h$-versus- $\Delta t$ data with Eq. (B.1) reveals the magnitude of the drift rate present in the data $\left(C_{4}=-0.00782 \mathrm{~nm} / \mathrm{s}\right)$. Subtracting the now-known drift from the raw data, we obtain refined data shown in Fig. B.1(c), which after analysis gives an $\dot{\varepsilon}$-versus- $p$ curve that is unaffected by drift [Fig. B.1(d)]. The resulting $\dot{\varepsilon}$-versus- $p$ curve displays power law behavior with a constant stress exponent $(n \sim 24)$ for all times. Fig. B.1(d) also includes the $\dot{\varepsilon}$-versus- $p$ 
curve that results from analysis, with Eq. (B.1), of the raw, drift-influenced data plotted in Fig. B.1(a). The drift-uncorrected $\dot{\varepsilon}$-versus- $p$ curve does not exhibit constant power law behavior; the extracted stress exponent increases with time from $n=24.4$ to $n=29.1$. Hence, the presence of drift in raw indentation data can give unrealistic stress exponents when the stress exponent is obtained from the $\log _{10}(\dot{\varepsilon})$-versus- $\log _{10}(p)$ curve slope at long times. Proper graphical determination of the stress exponent can be made after following the steps outlined in Fig. B.1 for computing drift-free $\dot{\varepsilon}$-versus- $p$ curves. With less effort, however, the physically meaningful stress exponent may be extracted, non-graphically, by inspection of Eq. (B.1) after it is fit to the raw $h$-versus- $\Delta t$ data. We compare Eqs. (9) and (10), note that $C_{3}=1 /(2 n)$, and then use this simple relationship to convert $C_{3}$ from the fit Eq. (B.1) to $n$. When applied to the data in Fig. B.1(a), this approach gives a stress exponents of $n=23.8$, which nearly matches the value obtained from graphical slope measurement $(n \sim 24)$ of drift-subtracted data.

One may suppose that it would be easiest to simply subtract drift from the raw data using the conventional approach to nanoindentation thermal drift removal. The standard technique for determining the sample drift rate involves measuring drift during a load hold, at a small load, either prior to or following the creep measurement. However, with viscoplastic samples, precisely measuring the thermal drift rate in such a manner may be difficult, as some materials creep or recovery may obscure the true magnitude of the thermal drift rate. It is thus likely that some drift_ - perhaps linear with time - inexorably remains in nominally drift-corrected data.

\section{References}

[1] J.M. Tarascon, M. Armand, Nature 414 (2001) 359-367.

[2] B. Scrosati, Electrochim. Acta 45 (2000) 2461-2466. 
[3] V. Etacheri, R. Marom, R. Elazari, G. Salitra, D. Aurbach, Energy Environ. Sci. 4 (2011) $3243-3262$.

[4] C.K. Chan, H. Peng, G. Liu, K. McIlwrath, X.F. Zhang, R.A. Huggins, Y. Cui, Nature Nanotech. 3 (2008) 31-35.

[5] N. Liu, Z. Lu, J. Zhao, M.T. McDowell, H.W. Lee, W. Zhao, Y. Cui, Nature Nanotech. 9 (2014) 187-192.

[6] H. Wu, G. Chan, J.W. Choi, I. Ryu, Y. Yao, M.T. McDowell, S.W. Lee, A. Jackson, Y. Yang, L. Hu, Y. Cui, Nature Nanotech. 7 (2012) 310-315.

[7] H. Ma, F. Cheng, J. Chen, J. Zhao, C. Li, Z. Tao, J. Liang, Adv. Mater. 19 (2007) 4067-4070. [8] Y. Yao, M.T. McDowell, I. Ryu, H. Wu, N. Liu, L. Hu, W.D. Nix, Y. Cui, Nano Lett. 11 (2011) 2949-2954.

[9] T. Song, J. Xia, J.H. Lee, D.H. Lee, M.S. Kwon, J.M. Choi, J. Wu, S.K. Doo, H. Chang, W.I. Park, D.S. Zang, H. Kim, Y. Huang, K.C. Hwang, J.A. Rogers, U. Paik, Nano Lett. 10 (2010) 1710-1716.

[10] M. Ge, J. Rong, X. Fang, C. Zhou, Nano Lett. 12 (2012) 2318-2323.

[11] M.N. Obrovac, L. Christensen, Electrochem. Solid-State Lett. 7 (2004) A93-A96.

[12] M. Pharr, K. Zhao, X. Wang, Z. Suo, J.J. Vlassak, Nano Lett. 12 (2012) 5039-5047.

[13] X.H. Liu, J.W. Wang, S. Huang, F. Fan, X. Huang, Y. Liu, S. Krylyuk, J. Yoo, S.A. Dayeh, A.V. Davydov, S.X. Mao, S.T. Picraux, S. Zhang, J. Li, T. Zhu, J.Y. Huang, Nature Nanotech. 7 (2012) 749-756.

[14] J.W. Wang, Y. He, F. Fan, X.H. Liu, S. Xia, Y. Liu, C.T. Harris, H. Li, J.Y. Huang, S.X. Mao, T. Zhu, Nano Lett. 13 (2013) 709-715.

[15] K. Zhao, M. Pharr, S. Cai, J.J. Vlassak, Z. Suo, J. Am. Ceram. Soc. 94 (2011) S226-S235. 
[16] H. Yang, S. Huang, X. Huang, F. Fan, W. Liang, X.H. Liu, L.Q. Chen, J.Y. Huang, J. Li, T. Zhu, S. Zhang, Nano Lett. 12 (2012) 1953-1958.

[17] S. Huang, F. Fan, J. Li, S. Zhang, T. Zhu, Acta. Mater. 61 (2013) 4354-4364.

[18] L.A. Berla, S.W. Lee, I. Ryu, Y. Cui, W.D. Nix, J. Power Sources 258 (2014) 253-259.

[19] I. Ryu, S.W. Lee, H. Gao, Y. Cui, W.D. Nix, J. Power Sources 255 (2014) 274-282.

[20] V.A. Sethuraman, M.J. Chon, M. Shimshak, V. Srinivasan, P.R. Guduru, J. Power Sources. 195 (2010) 5062-5066.

[21] V.A. Sethuraman, M.J. Chon, M. Shimshak, N. Van Winkle, P.R. Guduru, Electrochem. Comm. 12 (2010) 1614-1617.

[22] S.P.V. Nadimpalli, V.A. Sethuraman, G. Bucci, V. Srinivasan, A.F. Bower, P.R. Guduru, J. Electrochem. Soc. 160 (2013) A1885-A1893.

[23] M. Pharr, Z. Suo, J.J. Vlassak, Nano Lett. 13 (2013) 5570-5577.

[24] A. Kushima, J.Y. Huang, J. Li, ACS Nano. 6 (2012) 9425-9432.

[25] Z. Zeng, N. Liu, Q. Zeng, Y. Ding, S. Qu, Y. Cui, W.L. Mao, J. Power Sources. 242 (2013) $732-735$.

[26] J.B. Ratchford, B.E. Schuster, B.A. Crawford, C.A. Lundgren, J.L. Allen, J. Wolfenstine, J. Power Sources. 196 (2011) 7747-7749.

[27] J.B. Ratchford, B.A. Crawford, J. Wolfenstine, J.L. Allen, C.A. Lundgren, J. Power Sources. 211 (2012) 1-3.

[28] B. Hertzberg, J. Benson, G. Yushin, Electrochem. Comm. 13 (2011) 818-821.

[29] W.M. Robertson, D.J. Montgomery, Phys. Rev. 117 (1960) 440-442.

[30] S. Tariq, K. Ammigan, P. Hurh, R. Schultz, P. Liu, J. Shang, Proc. 2003 Part. Accel. Conf. 3 (2003) 1452-1454. 
[31] V.B. Shenoy, P. Johari, Y. Qi, J. Power Sources 195 (2010) 6825-6830.

[32] F. Fan, S. Huang, H. Yang, M. Raju, D. Datta, V.B. Shenoy, A.C.T. van Duin, S. Zhang, T. Zhu, Modelling Simul. Mater. Sci. Eng. 21 (2013) 074002.

[33] V.L. Chevrier, J.W. Zwanziger, J.R. Dahn, Can. J. Phys. 87 (2009) 625-632.

[34] Z. Cui, F. Gao, Z. Cui, J. Qu, J. Power Sources 207 (2012) 150-159.

[35] K. Zhao, W.L. Wang, J. Gregoire, M. Pharr, Z. Suo, J.J. Vlassak, E. Kaxiras, Nano Lett. 11 (2011) 2962-2967.

[36] H. Kim, C.Y. Chou, J.G. Ekerdt, G.S. Hwang, J. Phys. Chem. C 115 (2011) 2514-2521.

[37] J. Moon, K. Cho, M. Cho, Int. J. Prec. Eng. Man. 13 (2012) 1191-1197.

[38] G. Bucci, S.P.V Nadimpalli, V.A. Sethuraman, A.F. Bower, P.R. Guduru, J. Mech. Phys. Solids 62 (2014) 276-294.

[39] P. Danesh, B. Pantchev, J. Wiezorek, B. Schmidt, D. Grambole, Appl. Phys. A 102 (2011) 131-135.

[40] V. Kulikovsky, V. Vorlíček, P. Boháč, M. Stranyánek, R. Čtvrtlík, A. Kurdyumov, Thin Films 516 (2008) 5368-5375.

[41] V. Maier, B. Merle, M. Göken, K. Durst, J. Mater. Res. 28 (2013) 1177-1188.

[42] T.D. Hatchard, J.R. Dahn, J. Electrochem. Soc. 151 (2004) A838-A842.

[43] J.B. Puthoff, J.E. Jakes, H. Cao, D.S. Stone, J. Mater. Res. 24 (2014) 1279-1290.

[44] S.T. Boles, C.V. Thompson, O. Kraft, R. Monig, Appl. Phys. Lett. 103 (2013) 263906.

[45] H. Li, A.H.W. Ngan, J. Mater. Res. 19 (2004) 513-522.

[46] Y.J. Huang, Y.L. Chiu, J. Shen, J.J.J. Chen, J.F. Sun, J. Mater. Res. 24 (2009) 993-997.

[47] Y. Liu, I.K. Lin, X. Zhang, Mater. Sci. Eng. A 489 (2008) 294-301.

[48] C.L. Wang, M. Zhang, T.G. Nieh, J. Phys. D: Appl. Phys. 42 (2009) 115405. 
[49] L. Shen, W.C.D. Cheong, Y.L. Foo, Z.Chen, Mater. Sci. Eng. A 532 (2012) 505-510.

[50] Z.H. Cao, P.Y. Li, X.K. Meng, Mater. Sci. Eng. A 516 (2009) 253-258.

[51] Z.H. Cao, H.M. Lu, X.K. Meng, A.H.W. Ngan, J. Appl. Phys. 105 (2009) 083521.

[52] D. Peykov, E. Martin, R.R. Chromik, R. Gauvin, M. Trudeau, J. Mater. Sci. 47 (2012)

7189-7200.

[53] C. Su, E.G. Herbert, S. Sohn, J.A. LaManna, W.C. Oliver, G.M. Pharr, J. Mech. Phys. Solids 61 (2013) 517-536.

\section{Figure Captions}

Fig. 1. Characterization of silicon films used for indentation studies. (a) X-ray diffraction scan confirming that the as-sputtered silicon films are amorphous. Only peaks corresponding to the underlying molybdenum substrate are seen. (b) A plot showing electrochemical data from full lithiation of one amorphous silicon film. Lithiation voltage versus $\mathrm{Li} / \mathrm{Li}^{+}$(solid) and current (dashed) are plotted versus time.

Fig. 2. Scanning electron microscopy image $\left(52^{\circ}\right.$ sample tilt $)$ of a lithiated silicon film. Blistering at the microscale is observed.

Fig. 3. Results from continuous stiffness measurement (CSM) nanoindentation experiments performed on amorphous silicon, electrochemically formed lithium/silicon alloys of various compositions, and pure lithium. (a) The composite film/substrate Young's moduli from all twenty four indents made on fully lithiated silicon $\left(x_{L i}=0.79\right)$ are presented as a function of contact depth. The averaged modulus is also shown. (b) The composite Young's modulus, after 
data averaging, is plotted versus contact depth for each sample. (c) The extrapolation procedure used for extraction of film moduli is outlined on fully lithiated silicon data. (d) A plot of the composite indentation hardness, after data averaging, as a function of contact depth for all indented samples.

Fig. 4. Plots of film Young's modulus (a) and film hardness (b), free of substrate influence, versus lithium fraction. Data from our experiments ("Berla et al.") are represented with filled triangles. Other available published quasi-static nanoindentation data are included for comparison. The fully lithiated silicon composition is demarcated with a vertical dashed line.

Fig. 5. Normalized stiffness change (\%) is shown for both heavily lithiated silicon and unlithiated, amorphous silicon. Stiffness data are recorded using the CSM indentation technique during a $600 \mathrm{~s}$ constant load hold. The data demonstrate that lithiated silicon creeps much more rapidly than unlithiated silicon at a similar nominal indentation pressure.

Fig. 6. The creep analysis and corresponding results from quasi-static indentation creep experiments on heavily lithiated silicon. Depicted in (a) are small-time data $(t<20 \mathrm{~s})$ from one representative load hold $(15 \mathrm{mN})$. Eq. (2) fits closely to data at such times. Units of the provided fitting constants are such that displacements are in nanometers and time is in seconds. (b) In this same time range, the calculated strain rate is plotted versus pressure for all nine creep tests.

Fig. 7. The creep analysis and corresponding results from continuous stiffness measurement (CSM) indentation creep experiments on heavily lithiated silicon samples. (a) Displacements 
extracted from contact stiffnesses, measured during load hold $(\sim 16.5 \mathrm{mN})$ on one sample, are plotted versus time, and (b) depicts the calculated strain rate as a function of pressure. (c) Displacements extracted from contact stiffnesses, measured during a load hold $(\sim 5.7 \mathrm{mN})$ on another sample, are plotted versus time. The load is here held at a reduced indentation pressure by partially unloading from $\sim 16 \mathrm{mN}$ to $\sim 5.7 \mathrm{mN}$. (d) The calculated strain rate is plotted versus indentation pressure for this partially unloaded creep study. In (a) and (c), units of the provided fitting constants are such that displacements are in nanometers and time is in seconds.

Fig. 8. Outcome of a quasi-static nanoindentation recovery test. (a) The utilized load-versus-time profile is illustrated. A constant indentation load is applied for creep $(15 \mathrm{mN})$ and recovery $(0.68$ $\mathrm{mN}$ ) monitoring, and in each case the load is held for 600 s. The load-ramp and load-drop segments are each $10 \mathrm{~s}$ in duration. (b) A displacement-versus-time curve from one representative experiment.

Fig. B.1. Creep analysis—not limited to small times—applied to data from one representative quasi-static indentation creep test on heavily lithiated silicon. (a) Raw displacement data are plotted versus time for the entire duration of the constant-load hold (300 s). In (b), the same data are again presented, and Eq. (B.1) is shown to provide a good fit to the data. Units of the listed fitting constants are such that displacements are in nanometers and time is in seconds. (c) Drift $\left(C_{4}=0.00782 \mathrm{~nm} / \mathrm{s}\right)$ is subtracted from the data in (b), and the refined data are shown. (d) The strain rate-versus pressure curve computed from the raw displacement data is plotted alongside that calculated from the drift-subtracted data. 

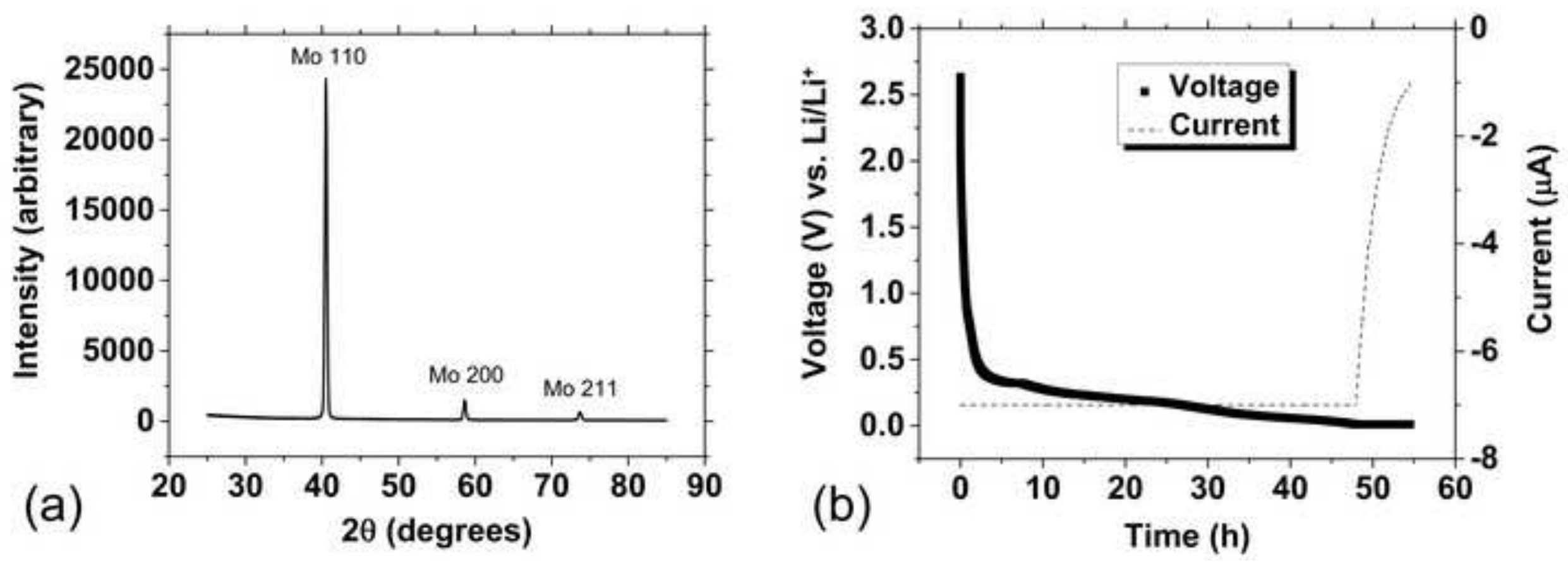
Figure2 

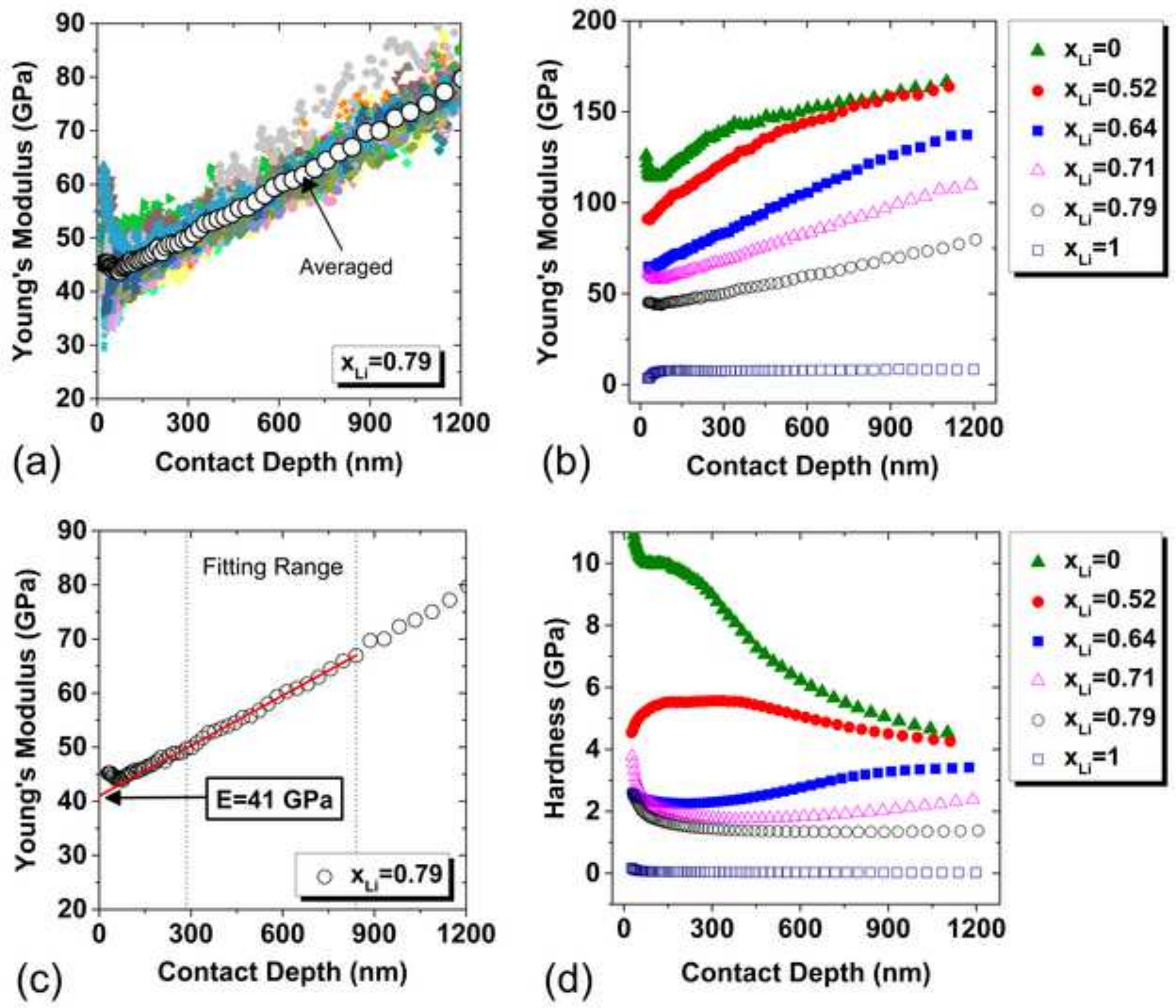

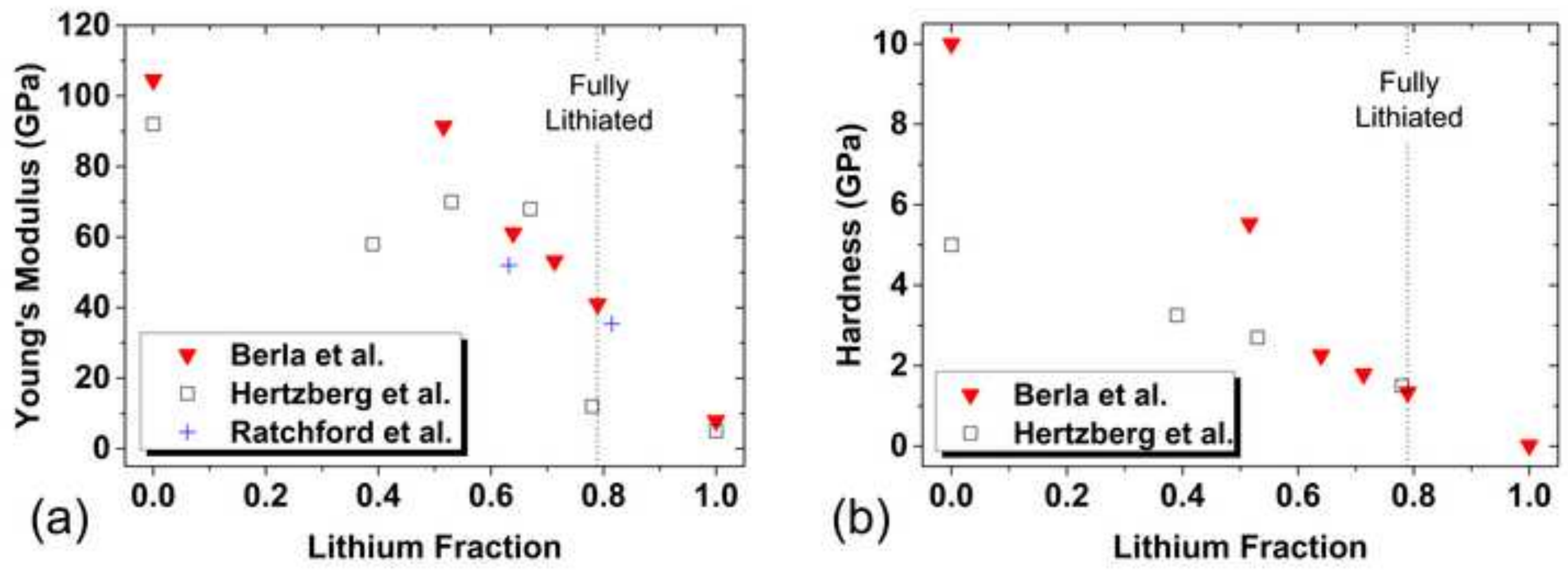


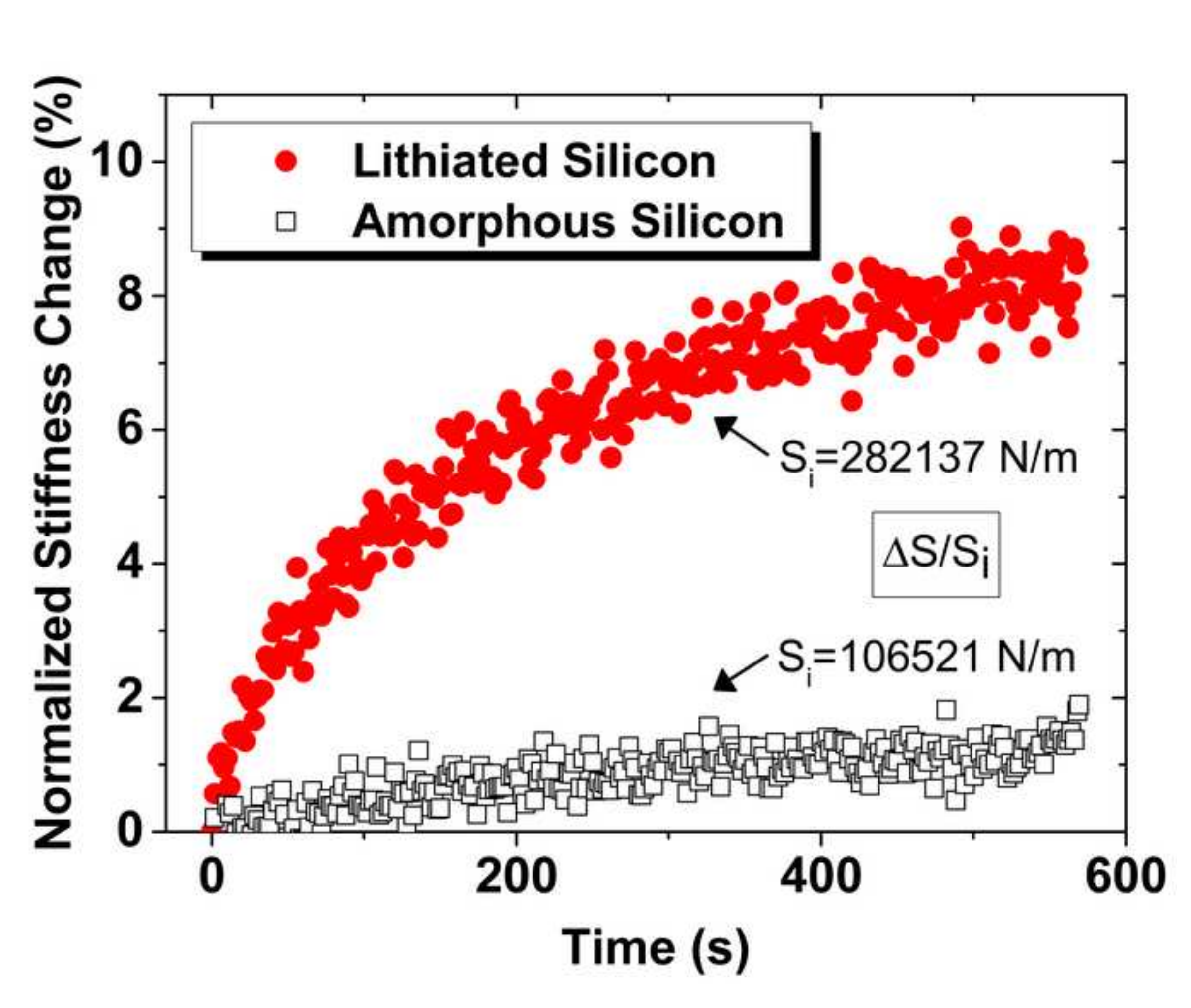

.

Figu
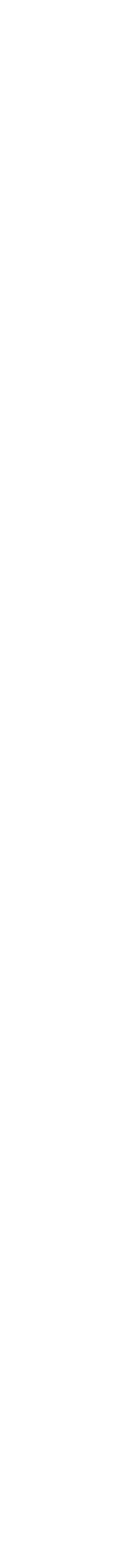

.

.


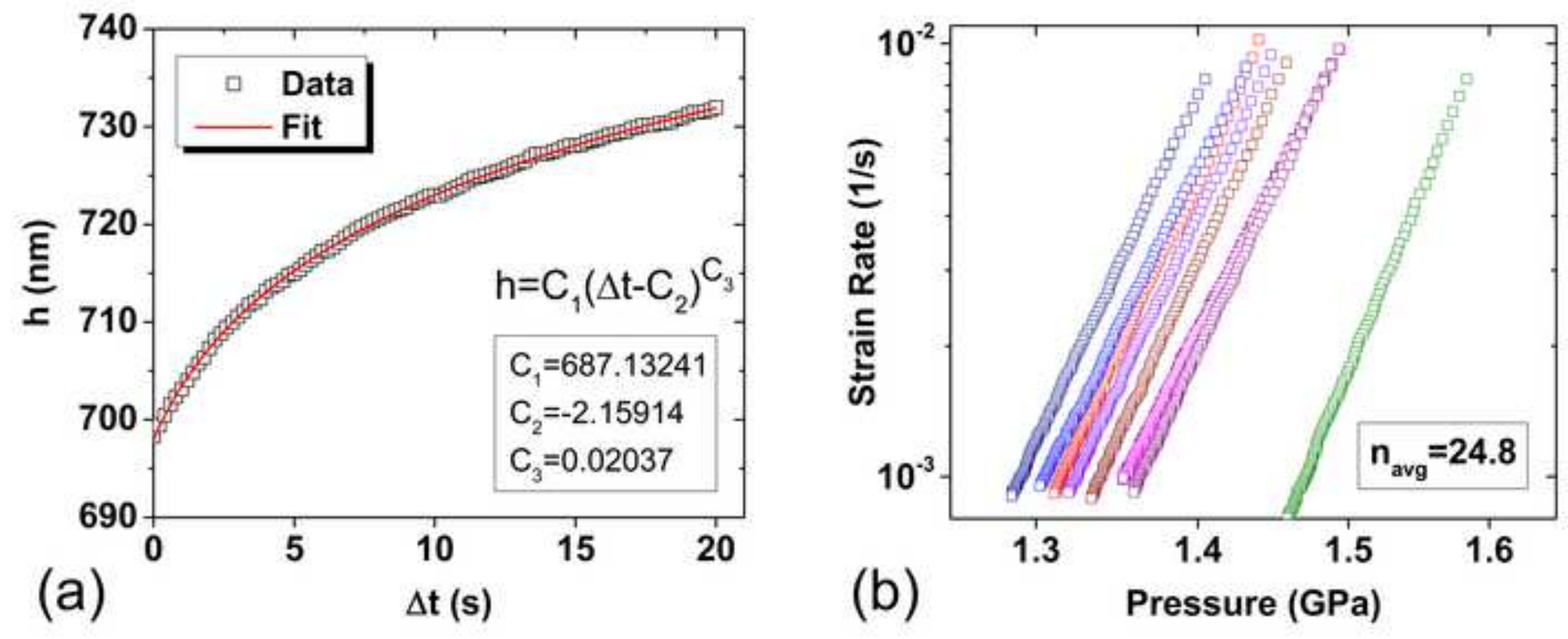


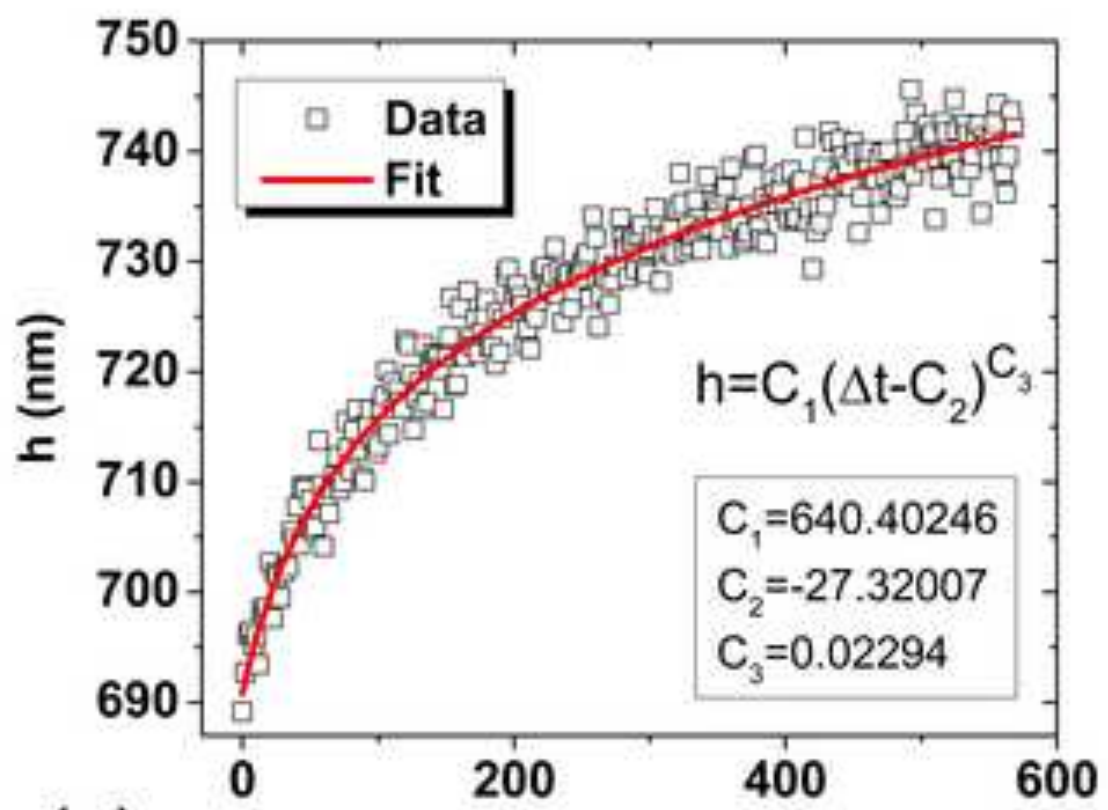

(a)

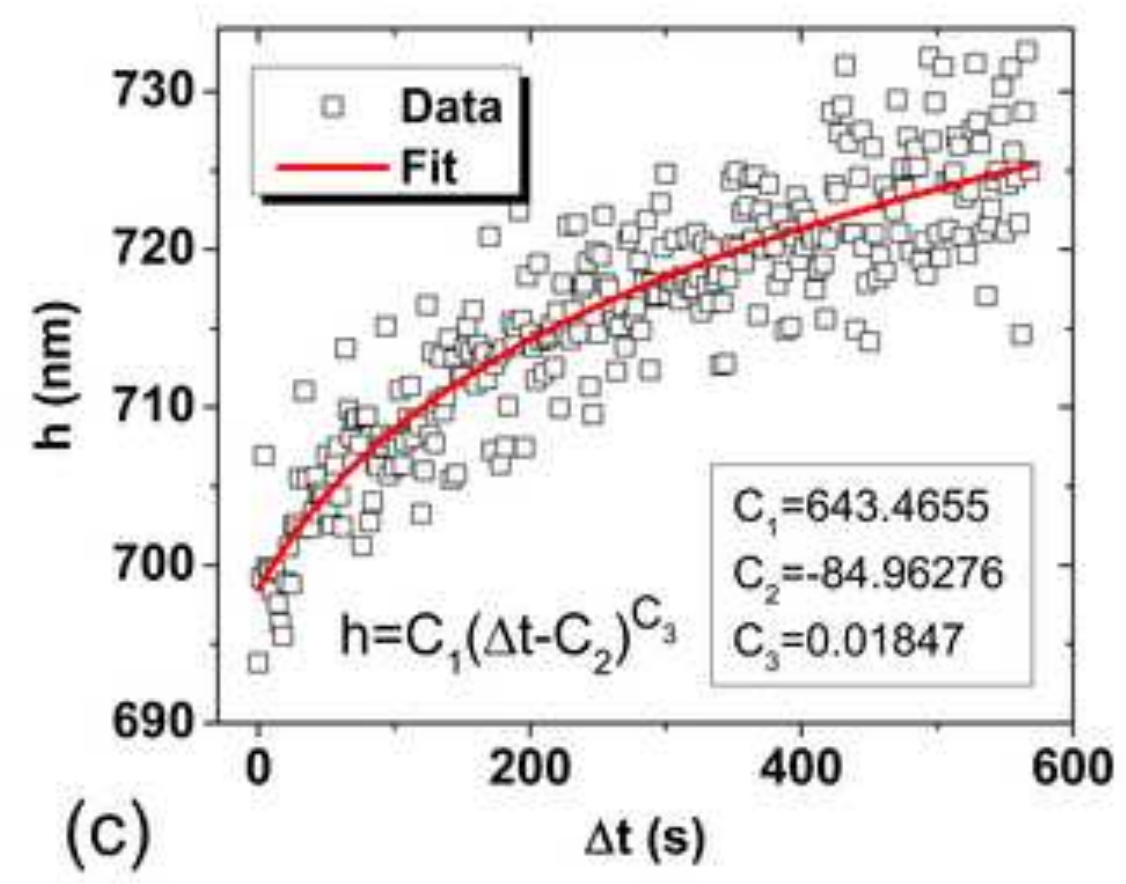

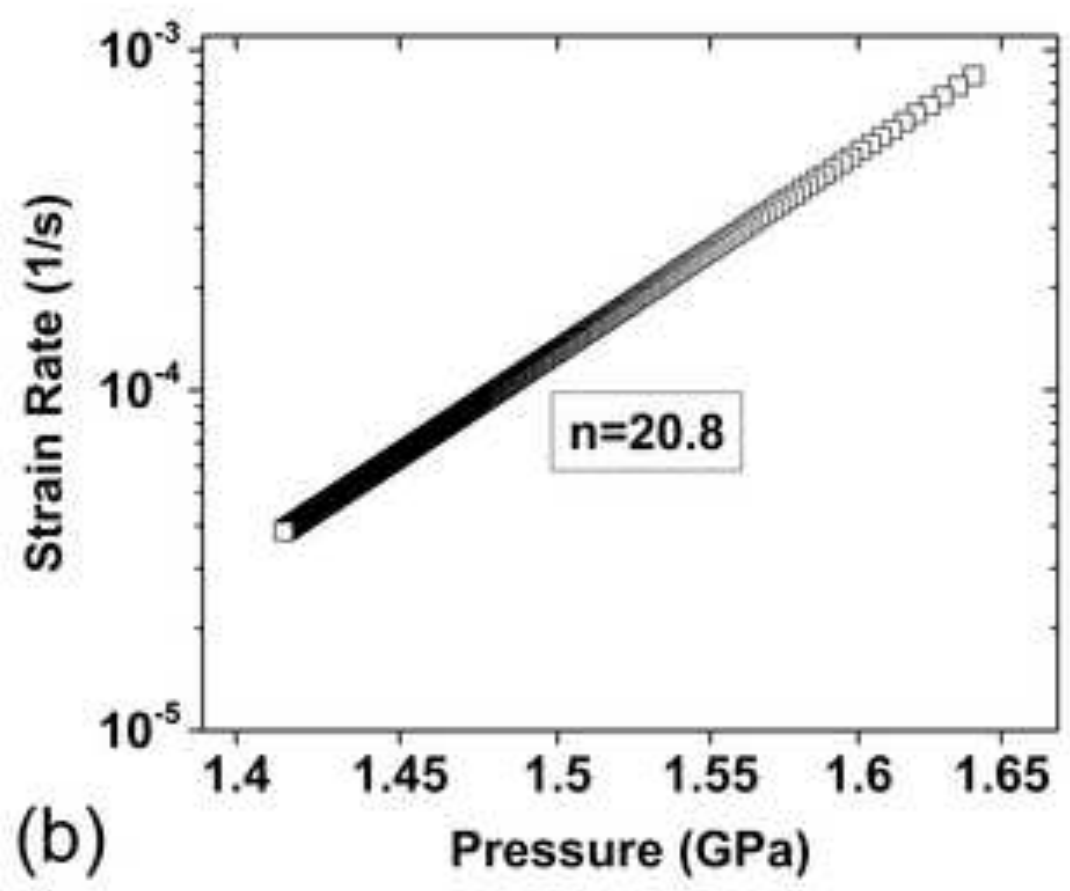

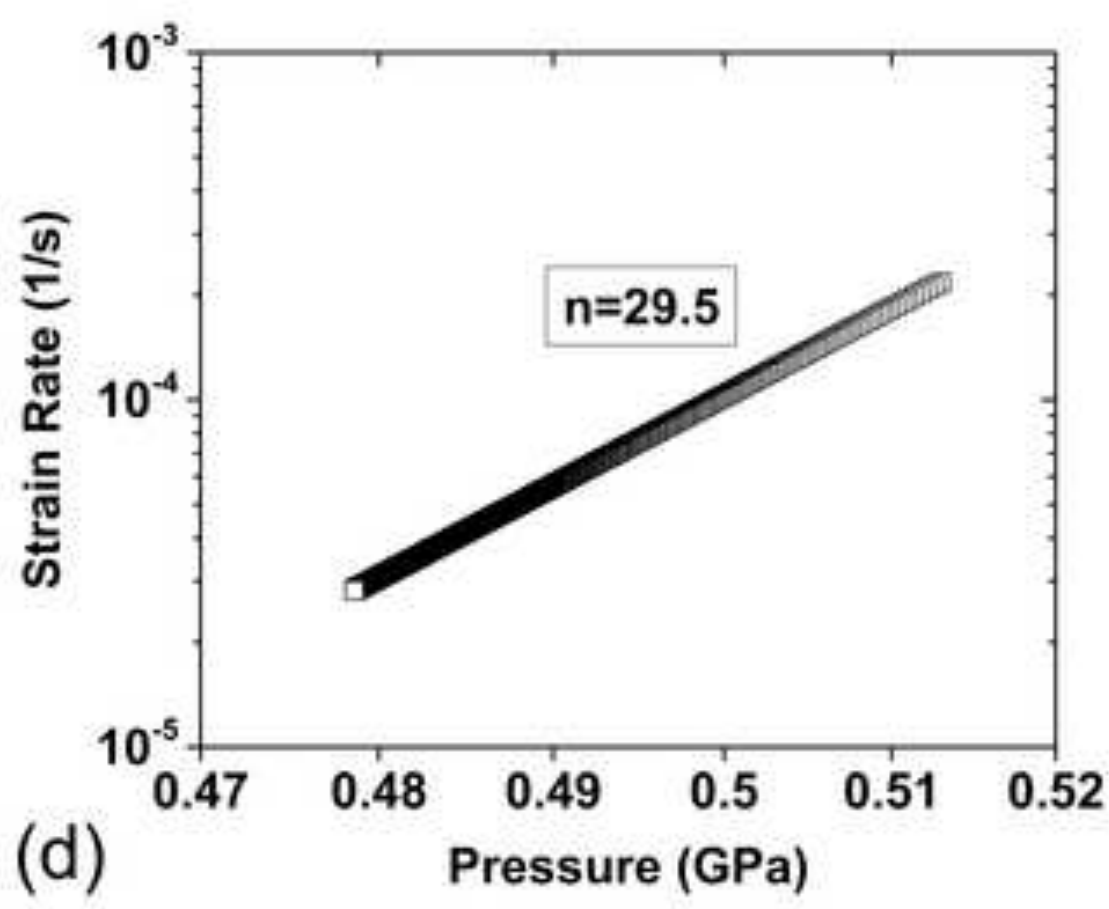



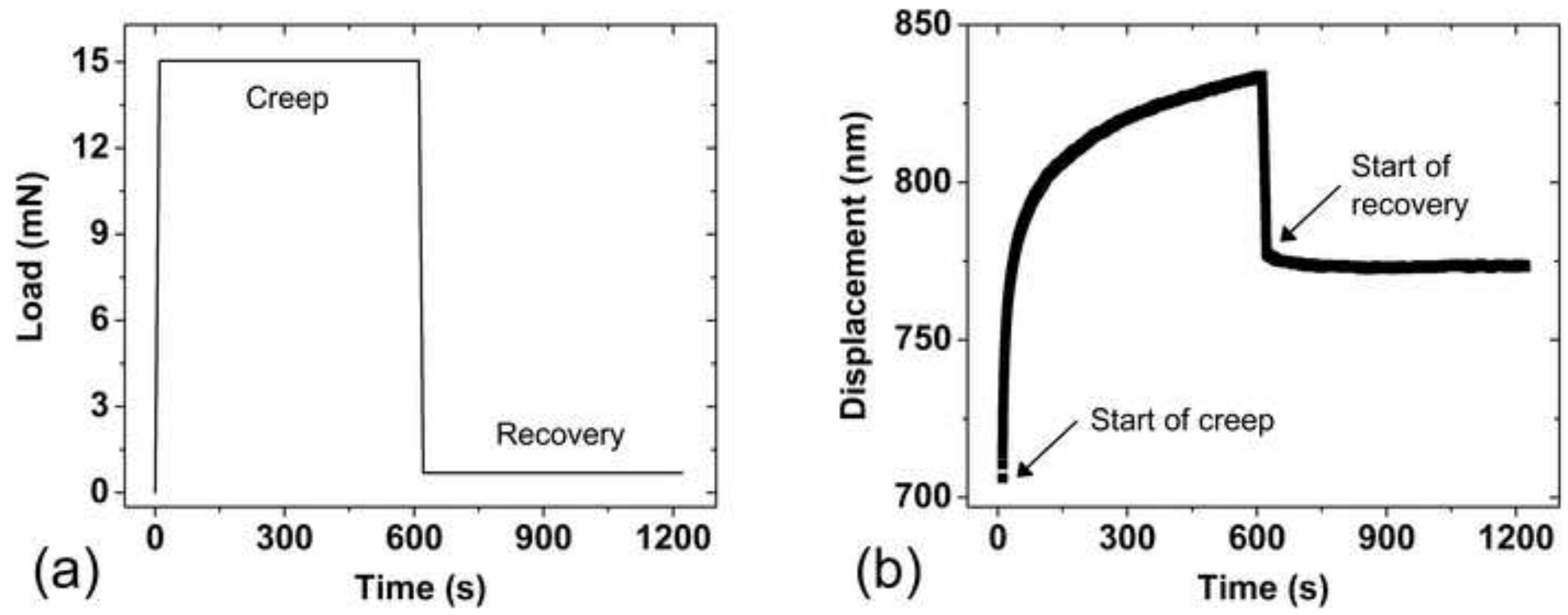

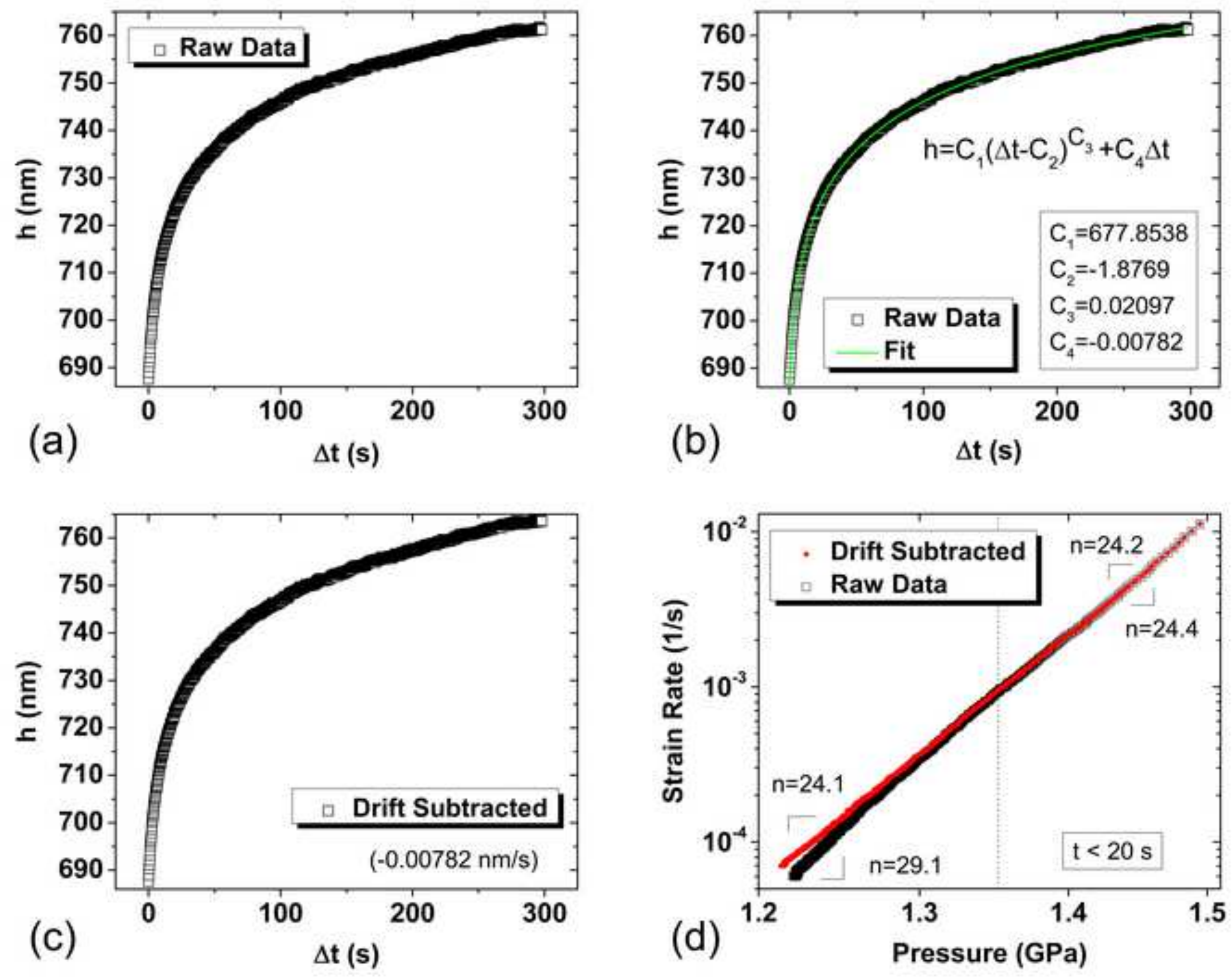